\title{
Liquid biopsy in pancreatic cancer
}

\begin{abstract}
Pancreatic cancer is the 9 th most frequent cancer but it stood as the 4 th cause of cancer death in 2016 and it is one of the few cancers in which mortality keeps increasing. Due to the inherent difficulty of this cancer for an early diagnosis and its relatively difficult access for performing a biopsy, increasing efforts are being done for developing liquid biopsy in PDA. While our knowledge of the biology of PDA has notably improved in recent years, the development of modern targeted therapies needs to be coupled to the existence of reliable biomarkers for monitoring. Different tumor constituents such as circulating tumor cells (CTCs), circulating tumor DNA (ctDNA) and extracellular vesicles (EXV), are shed into the circulation by tumors and can be detected in the peripheral blood of patients with cancer. This review will summarize the advances that have recently been made in the field of liquid biopsy for pancreatic cancer.
\end{abstract}

Special Issue - 2018

\section{Santiago Cabezas-Camarero}

Medical Oncology Department, Hospital Clínico Universitario San Carlos, Spain

Correspondence: Santiago Cabezas Camarero, Medical Oncology Department, Hospital Clínico Universitario San Carlos, Paseo del Prof. Martín Lagos S/N , 28040 Madrid, Spain, Tel +34 9l 3302667, FAX +34 9l 3303540 Email santicc8I@gmail.com

Received: April 23, 2017 | Published: September 19, 2018

Keywords: pancreatic cancer, pancreas, liquid biopsy, circulating tumor cells, circulating tumor dna, extracellular vesicles, exosomes, ctc, ctdna, digital pcr, peripheral blood

Abbreviations: PDA, pancreatic ductal adenocarcinoma; CTCs, circulating tumor cells; ctDNA, circulating tumor DNA; EXV, extracellular vesicles; NGS, next generation sequencing; OS, overall survival; EpCAM, epithelial-cell adhesion molecule; NE-iFISH, negative enrichment method coupled to immunofluorescence in situ hybridization; ddPCR, digital droplet PCR; TEX, tumor exosomes; MAF, mutant allele frequency

\section{Introduction}

Pancreatic ductal adenocarcinoma (PDA) constitutes one of the main causes of cancer death in the estern World. In the USA it was estimated that in 2016 it was the 9th most frequent cancer. Despite its moderate incidence, it stood as the 4th cause of cancer death and it is expected to be the second deadliest cancer by $2030 .{ }^{1}$ This discouraging data are explained, at least in part, by the difficulty for an early diagnosis due to the absence of symptoms in early stages and low yield of imaging tests, and by the poor therapeutic results in more advanced stages. Indeed, median survival in patients with metastatic disease is less than a year with current chemotherapy regimens. ${ }^{2,3}$ While our knowledge of the biology of PDA has notably improved in recent years, the development of effective therapies is hampered by the relative inaccessibility of this tumor to traditional biopsy. Therefore, increasing efforts are being done to refine liquid biopsy technologies in PDA. Different tumor constituents such as circulating tumor cells (CTCs), circulating tumor DNA (ctDNA) and extracellular vesicles (EXV) can be detected in the peripheral blood of patients with cancer, and also in patients with PDA. Most studies of liquid biopsy have focused in applying it as a prognostic biomarker or as a tool for monitoring response to treatment. This review will summarize the most relevant advances in liquid biopsy for patients with pancreatic cancer.

\section{Circulating tumor cells}

Several research groups have provided interesting data in the past few years in the field of CTCs in pancreatic cancer. However, CTC technologies are hampered by a still low-to-moderate sensitivity of detection. While various studies have shown the prognostic value of CTCs in PDA, the still low isolation capacity of most CTC technologies, limits the amount of DNA and RNA that can be obtained for conducting molecular studies, and therefore their value as a predictive tool is debated. ${ }^{4,5}$ Nevertheless, the advent of digital PCR and Next Generation Sequencing (NGS) have improved the quality of molecular studies performed on CTCs, since they need very small amounts of sample. ${ }^{5}$ Kullemann et al., ${ }^{6}$ using a size-based detection method, found CTCs in 8 of 10 patients with localized PDA and in 10 out of 11 patients with locally advanced or metastatic disease. They studied the KRAS pG12V mutation in CTCs, and found it predicted a longer overall survival (OS) (19.4 vs 7.4 months, $\mathrm{P}=0.015$ ). Earl et al. ${ }^{7}$ used CellSearch ${ }^{\circledR}$, an EpCAM-based isolation method, and detected CTCs in 7 out of 35 patients ( 1 with localized disease and 6 with metastases). In this study the presence of CTCs associated with a worse prognosis (OS CTC + vs CTC: 88 vs 395 days, $\mathrm{P}=0.0108$ ). Sergeant et al. ${ }^{8}$ using a method based on negative depletion of CD45+ and CD34+ cells, detected the presence of CTCs in 6/10 patients after tumor resection. The authors demonstrated the expression in CTCs of a gene signature associated with cell migration. Khoja et al. ${ }^{9}$ compared the CellSearch ${ }^{\circledR}$ and ISET methods-the latter a sizebased detection method- in a prospective set of 54 patients with PDA in different stages. ISET detected CTCs in $93 \%$ of the patients comparing favorably with the $40 \%$ detection rate of CellSearch ${ }^{\circledR}$, and the median number of CTCs was also higher with ISET. Bidard and colleagues, ${ }^{10}$ using the CellSearch ${ }^{\circledR}$ method, found CTCs in $5 \%$ and $9 \%$ of 79 patients with locally-advanced PDA before, and after 2 months of chemotherapy, respectively, and their presence associated with a worse prognosis.

Dotan et al. ${ }^{11}$ used CellSearch ${ }^{\circledR}$ in a prospective series of 48 patients with metastatic PDA, and detected CTCs in $48 \%$ of them. They observed a worse prognosis in patients who's CTCs expressed MUC-1, a transmembrane glycoprotein related to epithelial to mesenchymal transition. Finally, Xu et al. ${ }^{12}$ used a negative enrichment method coupled to immunofluorescence in situ hybridization (NEiFISH) of chromosome 8 to detect CTCs. This method was able to detect CTCs in $90 \%$ of 40 patients in different disease stages, and they established $\geq 3$ triploid CTCs as the cut-off predicting a worse 
1 -year OS $(\mathrm{P}=0.0279)$ and $\mathrm{OS}(\mathrm{P}=0.0188)$. They also showed that the presence of circulating tumor microembolus associated with a poor response to chemotherapy in stage IV disease.

The presence of CTC is generally associated with a worse prognosis, although there is no established cut-off with prognostic value in PDA. While CTC recovery improves at higher disease stages, specificity remains high with most CTC detection systems in all stages. To date, size-based and negative enrichment immunoaffinity methods have shown a greater isolation efficiency, with sensitivities ranging from $80-90 \%$ compared to $5-48 \%$ of standard EpCAM-based technologies. The former could, therefore, be useful for improving early diagnosis of PDA.

\section{Circulating tumor DNA}

Similar to CTCs, although to a lesser extent, the detection of ctDNA is hampered by assay sensitivity. Indeed, most solid results have appeared in the past few years with the use of digital PCR and NGS methods. The group of Däbritz et al. ${ }^{13}$ detected KRAS mutations in $36 \%$ of 56 patients with PDA using real-time PCR. Using direct sequencing, Chen et al. ${ }^{14}$ detected KRAS mutations in $33 \%$ of 91 patients, and they predicted a worse outcome in patients with unresectable disease. Bettegowda et al. ${ }^{15}$ used digital PCR in a large sample of 155 patients with PDA, detecting ctDNA in more than $75 \%$ of patients with advanced disease and in as much as $57 \%$ of patients with localized disease. The group by Earl et al. ${ }^{7}$ using digital PCR, detected ctDNA in 31 out of 35 patients with PDA, and found a mutation in KRAS in 8 of them, which associated with a worse prognosis (OS KRAS mutant vs KRAS wild-type: 66 vs 772 days, $\mathrm{P}=0.001$ ). Takai et al. ${ }^{16}$ in another large sample of 95 patients with PDA stages I-III and in 163 patients with stage IV disease, using digital droplet PCR (ddPCR) and targeted deep sequencing, identified potentially targetable mutations in ctDNA in $29.2 \%$ of the cases. Sausen et al. ${ }^{17}$ by means of ddPCR, identified ctDNA in $43 \%$ of 51 resected stage II PDAs. Besides, the presence of ctDNA after tumor resection predicted recurrence 6 months in advance of clinical recurrence. Finally, in a large series by Kinugasa et al. ${ }^{18}$ mutation in KRAS in ctDNA was detected in up to $62.6 \%$ of patients by ddPCR with a concordance of $77.3 \%$ with KRAS mutation status in solid tumor. While the mutation in KRAS in the primary tumor had not prognostic value, the presence of mutant KRAS in ctDNA was associated with a significantly shorter survival $(\mathrm{P}=0.006)$. Studies using digital PCR have demonstrated very high sensitivities, either in localized stages of PDA (up to $57 \%$ sensitivity) or in advanced stages (up to $88 \%$ sensitivity), for the detection of frequent mutations in DNA hot-spots such as those in KRAS. Indeed, KRAS-mutant ctDNA in PDA has been recurrently associated with a worse prognosis. Due to the high prevalence of KRAS mutations in PDA, it may also serve in early PDA detection and in therapy monitoring. ${ }^{19}$

\section{Extracellular vesicles}

EXV are membrane-bound vesicular components shed by cells into the peripheral circulation and other body fluids. They differ in size and molecular cargo, and can be classified as apoptotic bodies $(1000-5000 \mathrm{~nm})$, intermediate-sized microvesicles $(200-1000 \mathrm{~nm})$, and exosomes $(30-150 \mathrm{~nm}){ }^{20}$ The latter are a rich source of proteins, mRNA and DNA. Indeed, they have been recently recognized as the most important reservoir of ctDNA in plasma. ${ }^{21,22}$ Besides, mutations in DNA from tumor exosomes (TEX) consistently reflect the mutational status of their parental cells..$^{20,21,23}$ TEX has been studied in many solid tumors, including PDA. They can be easily obtained by differential ultracentrifugation and are identified by the expression of some membrane proteins known as tetraspanins (CD63, CD9, CD81). ${ }^{20}$ Recently, Melo et al. ${ }^{24}$ identified glipican-1, as a highlysensitive and specific marker of TEX derived from patients with PDA (100\% sensitivity and specificity), allowing to differentiate patients with PDA and with premalignant lesions from patients with benign pancreatic disease and healthy controls. As mentioned before, TEX is also shed to other body fluids, such as saliva. In a murine model of PDA, the presence of TEX was indirectly demonstrated in the saliva and they have already been detected in saliva from patients with lung cancer.25 Due to the advantages of studying TEX (easy acquisition, potential for molecular studies, moderate costs), there is a growing interest in TEX as a liquid biopsy tool for early cancer detection and treatment monitoring, particularly in PDA. Indeed, Alleson et al.$^{26}$ have recently shown results of a large study comparing KRAS mutation detection by digital PCR in ctDNA and in DNA obtained from TEX in patients with localized and advanced PDA. They found a sensitivity and specificity of $75.4 \%$ and $92.6 \%$, respectively, for the detection of mutant KRAS in TEX DNA. They also detected a higher percentage of mutant KRAS in TEX DNA from patients with early stage disease $(43.6 \%)$ than previously reported and observed that a higher mutant allele frequency (MAF) of KRAS in TEX DNA was associated with a poor prognosis in these patients. However, up to $20 \%$ of healthy controls showed mutant KRAS in TEX DNA, demonstrating the need for improving the specificity of exosome isolation. As mentioned earlier, the discovery of glipican-1 as a TEX-specific biomarker for PDA, may help to refine their role as a liquid biopsy tool. ${ }^{24}$

Although to date only a few studies have been reported using TEX in PDA, are a promising tool for liquid biopsy due to their easy acquisition with very high sensitivities (75-100\%) and specificities (92-100\%). They seem especially valuable in early cancer detection and for therapy monitoring due to their rich molecular cargo. However, their prognostic value has yet to be established.

\section{Conclusion}

Several tumor components such as circulating tumor cells, circulating tumor DNA and exosomes are shed into the peripheral circulation and can be detected in patients with pancreatic cancer. Although each detection technology has different applications, all of them may have great utility in pancreatic cancer diagnosis and in monitoring therapy. In the near future, liquid biopsy will certainly become a reality in the daily clinical practice of pancreatic cancer.

\section{Acknowledgements}

None.

\section{Conflict of interest}

Author declares that there is no conflict of interest.

\section{References}

1. Siegel RL, Miller KD, Jemal A. Cancer statistics, 2016. CA Cancer J Clin. 2016;66(1):7-30.

2. Conroy T, Desseigne F, Ychou M, et al. FOLFIRINOX versus gemcitabine for metastatic pancreatic cancer. NEngl JMed.2011;364(19):1817-1825.

3. Von Hoff DD, Ervin T, Arena FP, et al. Increased survival in pancreatic cancer with nab-paclitaxel plus gemcitabine. $N$ Engl $J$ Med. 2013;369(18):1691-1703. 
4. Ferreira MM, Ramani VC, Jeffrey SS. Circulating tumor cell technologies. Mol Oncol. 2016;10(3):374- 394.

5. Alix-Panabières C, Pantel K. Clinical applications of circulating tumor cells and circulating tumor DNA as liquid biopsy. Cancer. Disc 2016;6(5):479-491

6. Kulemann B, Liss AS, Warshaw AL, et al. KRAS mutations in pancreatic circulating tumor cells: a pilot study. Tumor Biol. 2016;37(6):7547-7554.

7. Earl J, Garcia Nieto S, Martinez-Avila JC, et al. Circulating tumor cells (Ctc) and kras mutant circulating free DNA (dfdna) detection in peripheral blood as biomarkers in patients diagnosed with exocrine pancreatic cancer. BMC Cancer. 2015;15:797.

8. Sergeant G, Eijsden R, Roskams T, et al. pancreatic cancer circulating tumor cells express a cell motility gene signature that predicts survival after surgery. BMC Cancer. 2012;12:527.

9. Khoja L, Backen A, Sloane R, et al. A pilot study to explore circulating tumor cells in pancreatic cancer as a novel biomarker. Br J Cancer. 2012;106(3):508-516.

10. Bidard FC, Huguet F, Louvet C, et al. Circulating tumor cells in locally advanced pancreatic adenocarcinoma: the ancillary CirCe 07 study to the LAP 07 trial. Ann Oncol. 2013;24(8):2057-2061.

11. Dotan E, Alpaugh K, Ruth K, et al. Prognostic significance of MUC-1 in circulating tumor cells in patients with metastatic pancreatic adenocarcinoma. Pancreas. 2016;45(8):1131-1135.

12. Xu Y, Qin T, Li J, et al. Detection of circulating tumor cells using negative enrichment immunofluorescence and an in situ hybridization system in pancreatic cancer. Int J Mol Sci. 2017;18(4): E622.

13. Dabritz J, Preston R, Hanfler J, et al. Follow-up study of K-ras mutational status in the plasma of patients with pancreatic cancer: correlation with clinical features and carbohydrate antigen CA 19-9. Pancreas. 2009;38(5):534-541

14. Bettegowda C, Sausen M, Leary RJ, et al. Detection of circulating tumor DNA in early- and late-stage human malignancies. Sci Transl Med. 2014;6(224):224ra24.

15. Chen $\mathrm{H}, \mathrm{Tu} \mathrm{H}$, Meng ZQ, et al. K-ras mutational status predicts poor prognosis in unresectable pancreatic cancer. Eur J Surg Oncol. 2010;36(7):657-662
16. Takai E, Totoki Y, Nakamura $\mathrm{H}$, et al. Clinical utility of circulating tumor DNA for molecular assessment in pancreatic cancer. Sci Rep. 2015;5:18425

17. Sausen M, Phallen J, Adleff V, et al. Clinical implications of genomic alterations in the tumor and circulation of pancreatic cancer patients. Nat Commun. 2015;6:7686.

18. Kinugasa H, Nouso K, Miyahara K, et al. Detection of K-ras gene mutation by liquid biopsy in patients with pancreatic cancer. Cancer. 2015;121(13):2271-2280.

19. Gao Y, Zhu Y, Yuan Z. Circulating tumor cells and circulating tumor DNA provide new insights into pancreatic cancer. Int J Med Sic. 2016;13(12):902-913.

20. Whiteside TL. the potential of tumor-derived exosomes for noninvasive cancer monitoring. Expert Rev Mol Diagn. 2015;15(10):1293-1310.

21. Kahlert C, Melo SA, Protopopov A, et al. Identification of double-stranded genomic DNA spanning all chromosomes with mutated KRAS and p53 DNA in the serum exosomes of patients with pancreatic cancer. $J$ Biol Chem. 2014;289(7):3869-3875.

22. Thakur BK, Zhang H, Becker A, et al. Double-stranded DNA in exosomes: a novel biomarker in cancer detection. Cell Res. 2014;24(6):766-769.

23. García-Romero N, Carrión-Navarro J, Esteban-Rubio S, et al. DNA sequences within glioma-derived extracellular vesicles can cross the intact blood-brain barrier and be detected in peripheral blood of patients. Oncotarget. 2017;8(1):1416-1428.

24. Melo SA, Luecke LB, Kahlert C, et al. Glypican-1 identifies cancer exosomes and detects early pancreatic cancer. Nature. 2015;523(7559):177-182.

25. Lau C, Kim Y, Chia D, et al. Role of pancreatic cancer-derived exosomes in salivary biomarker development. J Biol Chem. 2013;288(37):26888-26897.

26. Allenson K, Castillo J, San Lucas FA, et al. High prevalence of mutan KRAS in circulating exosome-derived DNA from early-stage pancreatic cancer patients. Ann Oncol. 2017;28(4): 741-747. 